\title{
Michelson Interferometer characterisation of noise reduction in DFB fibre lasers
}

\author{
Albert Canagasabey*a,c ${ }^{* a v i d ~ J o n e s}{ }^{b}$, David Mann ${ }^{\mathrm{b}}$, John Canning ${ }^{\mathrm{a}}$, Simon Fleming ${ }^{\mathrm{c}}$, \\ John Holdsworth ${ }^{\mathrm{d}}$ \\ anterdisciplinary Photonics Laboratories (iPL), School of Chemistry, University of \\ Sydney, 2006, NSW, Australia \\ ${ }^{b}$ Thales Underwater Systems, Rydalmere, 2116, NSW, Australia \\ 'Institute of Photonics and Optical Science (IPOS), School of Physics, University of \\ Sydney, 2006, NSW, Australia \\ ${ }^{\mathrm{d}}$ SMAPS, University of Newcastle, Callaghan, NSW 2308, Australia \\ *john.canning@sydney.edu.au
}

\begin{abstract}
A comparison is made between unpackaged and packaged distributed feedback (DFB) fibre lasers using the Michelson interferometer configuration for delayed self-heterodyne interferometery (MIDSHI) to ascertain the improvements to the external environmental noise, quantified by reductions in the Gaussian linewidth. Voigt fitting is used to extract and separate out the Lorentzian and Gaussian linewidth contributions and therefore the associated sources of noise. Significant improvements in the Gaussian linewidth were achieved as a result of significant reductions in the sensitivity of the DFB laser to external perturbations using packaging. However, a broadening of the laser Lorentzian linewidth was observed.
\end{abstract}

Keywords: Keywords: Heterodyne, DFB, Laser linewidth, Faraday rotation, Michelson interferometer, Gaussian noise, Lorentzian noise

\section{INTRODUCTION}

The inherently narrow linewidths of DFB fibre lasers make them attractive for applications such as optical communications, sensing and spectroscopy. One important application involving acoustic sensing are hydrophones based on an array of DFB fibre lasers are being developed to replace conventional sensors for sonar applications. They offer high reliability, low power budgets, insensitivity to electromagnetic interference and straightforward multiplexing. The high sensitivity of the DFB fibre laser to local strain is advantageous in detecting acoustic perturbations through frequency fluctuations of the laser which can be measured using interferometric techniques. This high degree of sensitivity is compounded by non-acoustic environmental perturbations which increase laser and system noise, reducing the overall sensitivity. Thus for hydrophone applications, the DFB fibre laser needs to be packaged appropriately to reduce its sensitivity to non-acoustic perturbations and to enhance its longitudinal strain sensitivity. This in turn dictates the type of design both for the laser and the package.

The application of the Schawlow-Townes formula to silica-based DFB fiber lasers would indicate that the linewidth can be $60 \mathrm{~Hz}$ or less [1]. However, in practice, the observed linewidths have been significantly greater, owing to increased phase noise which is often attributed to environmental perturbations and inherent thermal noise within the laser, both background and from non-radiative phonon-coupled decay within the gain medium which can often only be reduced by cooling to extremely cold temperatures or through the exploitation of molecular engineering to isolate relevant transitions. The two are coupled but their profiles can be distinct. DSHI has been employed for the 
measurement of laser linewidths since it was introduced in 1980 [2]. It has been particularly useful for the measurement of linewidths in semiconductor DFB lasers for which the most significant contribution to noise arises from spontaneous emission induced refractive index changes [3]. This noise is predominantly white in nature, enhanced through cavity resonance, for which the lineshape is Lorentzian. For DFB fiber lasers, however, the linewidth can be greatly influenced by other sources of noise which tend to be coloured in nature, characterised with a Gaussian line shape $[4,5]$.The presence of both sources of noise with dissimilar lineshapes complicates the analysis for the extraction of linewidth for the DFB fiber laser, but on the other hand, can be useful to identify sources of noise and their relative contributions. A convolution of the Lorentzian and Gaussian functions, such as the Voigt function, is typically required for linewidth extraction from interferometric measurements of fibre DFB laser outputs [6].

In practice, the contribution to the Gaussian linewidth has been primarily attributed to external thermal noise in the DFB cavity leading to refractive index fluctuations $[4,5]$. The main contributors to these thermal fluctuations have been identified to be ambient temperature fluctuations, self-heating, and fundamental temperature noise due to random diffusion of phonon-phonon coupled excitations [5] with acoustic fluctuations also being a potential contributor. Furthermore, self-heating of the DFB as a result of non-radiative dissipated pump power has also been identified as an additional source of potential noise capable of further broadening the linewidth $[7,8]$. An increase in linewidth, for both Lorentzian and Gaussian contributions, has been observed with increasing pump powers [8], attributed to temperature fluctuations caused by variations in absorbed pump energy. This apparent contravention of the Schawlow-Townes formula for the increase in the Lorentzian linewidth was argued as evidence that the broadening may not be entirely related to spontaneous emission alone [9]. The transition from the low pump power regime, where the noise predominantly derives from intrinsic thermal fluctuations, to the high power regime, where pump noise becomes significant, has also been observed in a direct measurement of the frequency noise of the DFB laser [10] Hence, there are a number of contributions to noise, both within the laser itself and from external sources including the pumping system, which cannot always be well quantified but can be qualitatively determined within the two profiles used to extract linewidth.

The direct measurement of linewidth using DSHI is typically carried out using a Mach-Zehnder interferometer (MZI) configuration employing conventional low-birefringence fiber. The MZI is prone to signal degradation as a result of fringe fading caused by random variations in the state of polarisation (SOP) of the two arms with respect to each other. This contribution is generally more problematic with increasing interferometer path length (necessary with decreasing laser linewidth) and can potentially cause signal (fringe) fading and result in significant measurement errors in the laser linewidth. The use of expensive high-birefringence fiber and components based on such fiber is typically ruled out given the very long delay lengths required for the DSHI and the associated complexities. Instead, a polarisation controller is inserted into one of the arms to match the SOP at the beginning of each measurement. Unfortunately, this is not ideal given the long measurement times required for the acquisition of the beat spectrum, as the SOP will drift due to environmental perturbations no matter how well shielded the setup. This drift in polarisation affects the observable interference and raises questions about the inherent noise in the MZI measurements. From an interferometery perspective, a solution is to use a Michelson interferometer (MI) which employs Faraday rotator mirrors (FRM) to compensate for birefringence effects, providing a polarisation-insensitive measurement technique that is immune to fringe fading.

In this paper we use DSHI in a MI configuration to measure and compare the linewidths of two identical DFB fibre lasers, one unpackaged and the other packaged to reduce environmental noise. A Voigt fit is used to extract the Gaussian and Lorentzian components of the linewidths for varying power levels. The linewidth enhancement as a result of packaging is quantified and explained in terms of the associated noise contributions. 


\section{EXPERIMENTS}

The DFB lasers used in these experiments were supplied by Redfern Optical Components fabricated under identical conditions by direct writing with $\mathrm{CW} 244 \mathrm{~nm}$ light from a frequency doubled $\mathrm{Ar}^{+}$laser into an $\mathrm{Er}^{3+}$ doped germanosilicate fiber. The phase-shifted grating is $55 \mathrm{~mm}$ long with an off-centre phase shift designed to preferentially direct single polarisation laser output from one side. One DFB laser was then re-coated and packaged.

The DFB fibre laser packaging was firstly designed to provide a robust housing which will survive the significant depth and handling requirements necessary for reliable sonar systems. The packaging topology provides both acoustic and vibration isolation within a low aspect ratio whilst still meeting strict density requirements. The packaging completely encapsulates the laser and provides strain relief and mechanical de-coupling of the lead-in fibre. Particular attention was paid to thermal considerations [11] and materials and adhesives were specifically selected to reduce the application of strain either during manufacture or due to temperature fluctuations during operation.

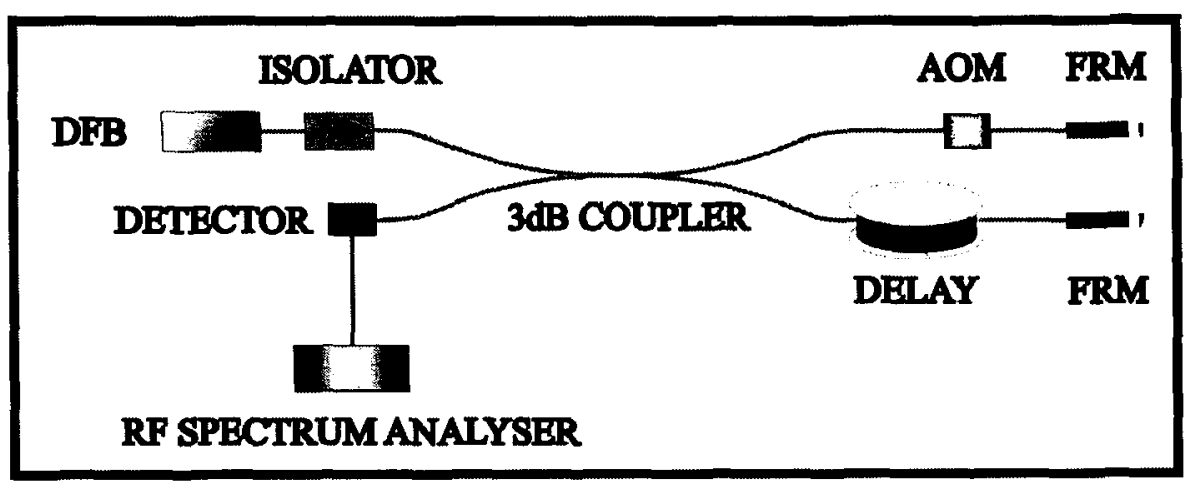

Figure 1. Delayed self-heterodyne Michelson interferometer (AOM: Acousto-Optic Modulator, FRM: Faraday Rotator Mirror)

A MI employing FRMs was constructed as shown in Figure 1. The DFB lasers and the interferometer were placed inside a soundproof enclosure which was in turn placed on an actively isolated electronic vibration isolation table. The light from the DFB laser is split, using a $2 \times 2$ coupler, into two arms, with one arm experiencing a $31 \mathrm{~km}$ delay, while in the other arm an acousto-optic modulator (AOM) is used to shift the frequency of light by $f=27.12 \mathrm{MHz}$ with each pass, which results in an overall shift of $f=$ $54.24 \mathrm{MHz}$. The arms are terminated with FRMs and the second input arm of the coupler is connected to a fast detector. With the return pass, the MI has an effective delay of $l=62 \mathrm{~km}$ and the resulting interference produces a beat spectrum at a frequency of $54.24 \mathrm{MHz}$.

The beat spectra resulting from the interference are viewed using a radio-frequency spectrum analyzer (RFSA) and an approximate value for the DFB laser coherence length can be simply determined from the RFSA trace; with a superimposed interference signal indicating coherence. When the $62 \mathrm{~km} \mathrm{MI}$ delay length the recombining light is no longer interfering and therefore incoherent - i.e. there is no observable interference signal. From this a laser linewidth below $1.5 \mathrm{kHz}$ can be expected.

The measured beat spectra from the DSHI technique contain linewidth broadening contributions from both white and coloured $(1 / f)$ noise sources which give rise to lineshape contributions that can be fitted with Lorentzian and Gaussian profiles, respectively [6]. The Voigt function, a convolution of these two contributions, can be fitted to the beat spectrum to separate out these components of the laser linewidth. This interpretation of noise dominated broadening is possible if the measured broadened linewidth is commensurate or larger than the natural laser linewidth. The Voigt profile is described by: 


$$
V=\frac{2 \ln 2}{\pi^{3 / 2}} \frac{w_{L}}{w_{G}} \cdot \int_{-\infty}^{+\infty} \frac{e^{-t^{2}}}{\left(\sqrt{\ln 2} \frac{w_{L}}{w_{G}}\right)^{2}+\left(\sqrt{4 \ln 2} \frac{f-f_{0}}{w_{G}}-t\right)^{2}} d t
$$

where, $w_{L}$ and $w_{G}$ represent the full-width-half-maximum (FWHM) of the Lorentzian and Gaussian lineshapes and $f$ is the frequency with $f_{0}$ being the centre frequency. The extracted Lorentzian linewidth is divided by a factor of 2 , and the Gaussian by a factor of $\sqrt{2}$ since the beat signal is a Voigt profile of two separate but identical spectral densities [12].

\section{RESULTS AND DISCUSSIONS}

The beat spectra for each DFB fibre laser was recorded with the RFSA for a range of laser output power levels, with each measurement averaged one hundred times. A total of ten measurements were made at each power level for each DFB laser to reduce uncertainty and ensure statistical reliability and reproducibility. The beat spectra, obtained at the resolution bandwidth setting of $10 \mathrm{~Hz}$ are shown for one $(285 \mu \mathrm{W})$ of the ten DFB laser power levels used are shown in Figure 2 (a) and (b) for the unpackaged and packaged DFB lasers, respectively. Each beat spectrum was fitted with the Voigt function to extract the Lorentzian and Gaussian contributions separately. Figure 2 (c) and (d) show examples of a Voigt fit of one measurement (at $285 \mu \mathrm{W}$ ) for unpackaged and packaged laser respectively.
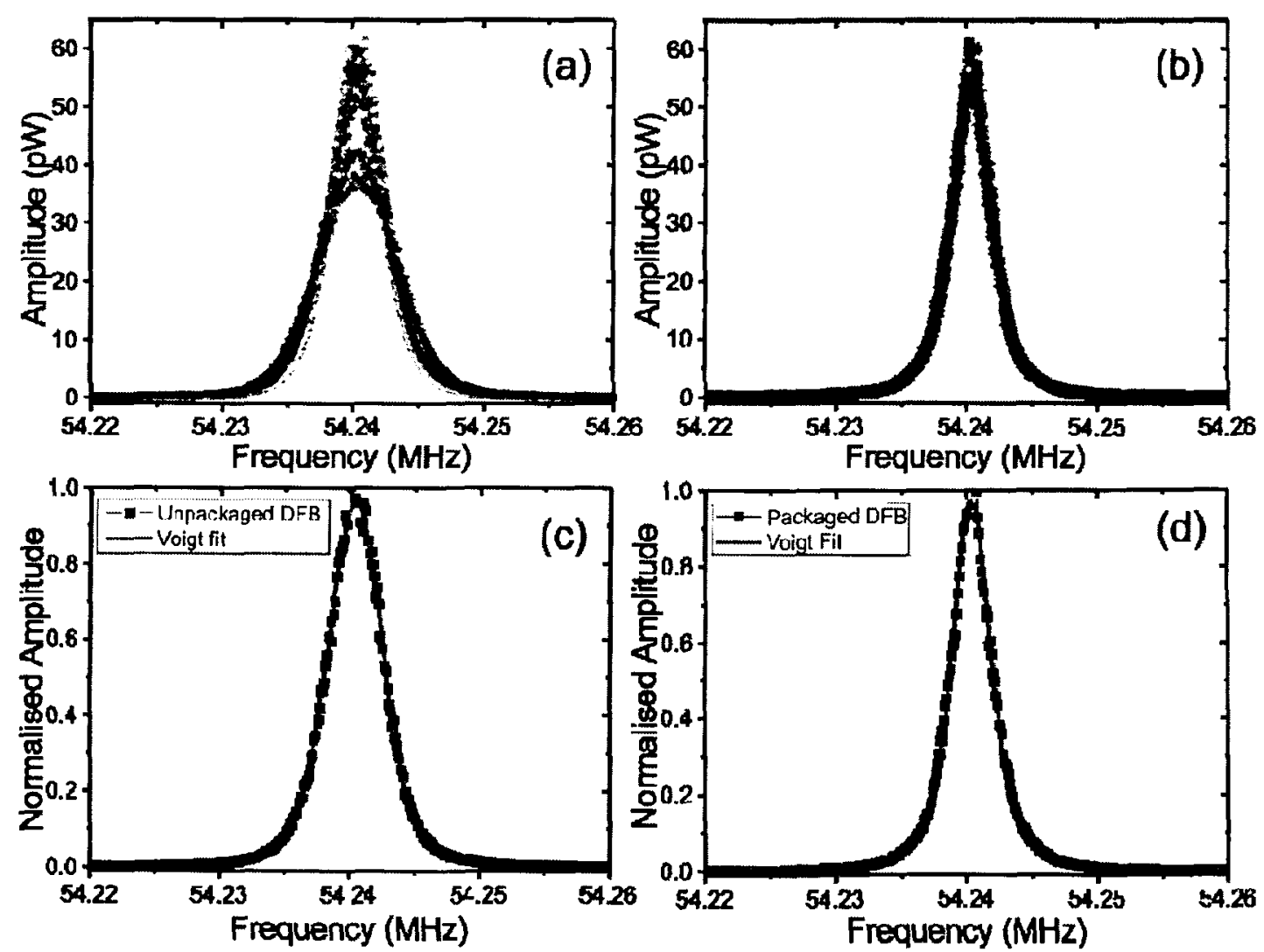

Figure 2 Beat spectra of 10 consecutive measurements each averaged 100 times for (a) unpackaged and (b) packaged DFB lasers at a DFB laser power of $285 \mu \mathrm{W}$. Voigt fitting for one of 10 acquired beat spectra for the DFB power of $285 \mu \mathrm{W}$ for the (c) unpackaged and (d) packaged DFB laser. 
From Figure 2 (b) the packaged DFB produces consistent, reproducible beat spectra. The unpackaged DFB laser, on the other hand, suffers from amplitude variations associated with random fluctuations in the Gaussian noise component caused by environmental perturbations. The use of the $\mathrm{Ml}$ configuration with FRMs for DSHI measurement rules out amplitude variations caused by fringe fading due to polarisation mismatch in the two arms [7]. Further, the DFB lasers were tested with the same components (couplers, FRMs, AOM and pump laser) to ensure identical test conditions. The Lorentzian and Gaussian linewidths extracted are plotted as functions of power in Figure 3 (a) and (b) for the unpackaged and packaged DFB lasers. The Lorentzian linewidth profiles for increasing DFB power are comparable for both DFBs, with the unpackaged DFB laser exhibiting higher measurement errors for some of the points. A $\sim 200 \mathrm{~Hz}$ lower linewidth is also observed at saturation for the unpackaged laser.

The differences between the packaged and unpackaged DFB are particularly evident for the Gaussian linewidth as seen in Figure 3(b), with the latter measuring values that were $>(1000-2500) \mathrm{Hz}$, with significantly greater errors in measurement. The packaged DFB on the other hand, shows a consistent Gaussian linewidth over the full power range with very little measurement error. The significantly greater contribution to the Gaussian linewidth in the case of the unpackaged DFB laser is expected given its susceptibility to environmental perturbations, such as variations in ambient temperature and acoustic noise. Self-heating as a result of poor heat dissipation could also contribute to the Gaussian linewidth for the unpackaged DFB laser. Inefficient heat transfer from the fibre to the environment could affect the stability of the output power, particularly with increasing pump powers. The placement of the DFB laser inside an enclosure placed on a vibration isolated table would undoubtedly have served to reduce environmental contributions to the noise the unpackaged DFB laser.

The measured differences in the Lorentzian linewidth are equally important - they show that there is broadening in the packaged DFB fibre laser. This can arise principally in two ways. One is where slight chirping of the grating in the package as a result of a strain gradient is introduced - this seems unlikely given that bonding is outside of the grating region and there is no pressure on the grating itself within the package. The second way is if the packaging has introduced a small amount of twist which can both slightly chirp the grating but also start to separate the polarisation eigenstates that are natural in the fibre but which have been compensated for during grating writing. Any applied tension from the package, through micro twisting, which exceeds that used during the grating writing process can lead to anisotropic phase shift difference that slightly displaces the two leading to separation. However, in the case of linearly written polarised compensated gratings this would usually lead to polarisation instability through polarisation hole burning and would be observable as amplitude fluctuations in the laser output on an oscilloscope on a sufficiently fast timescale - this is not observed. Therefore, since this is not observed we can make an educated guess as to the method of polarisation compensation in this laser - it is probably based on pre-twisting prior to inscription [13]. It is entirely feasible known the packaging routine in detail to potentially adjust the grating writing setup to remove this separation and further narrow the Lorentzian linewidth. 

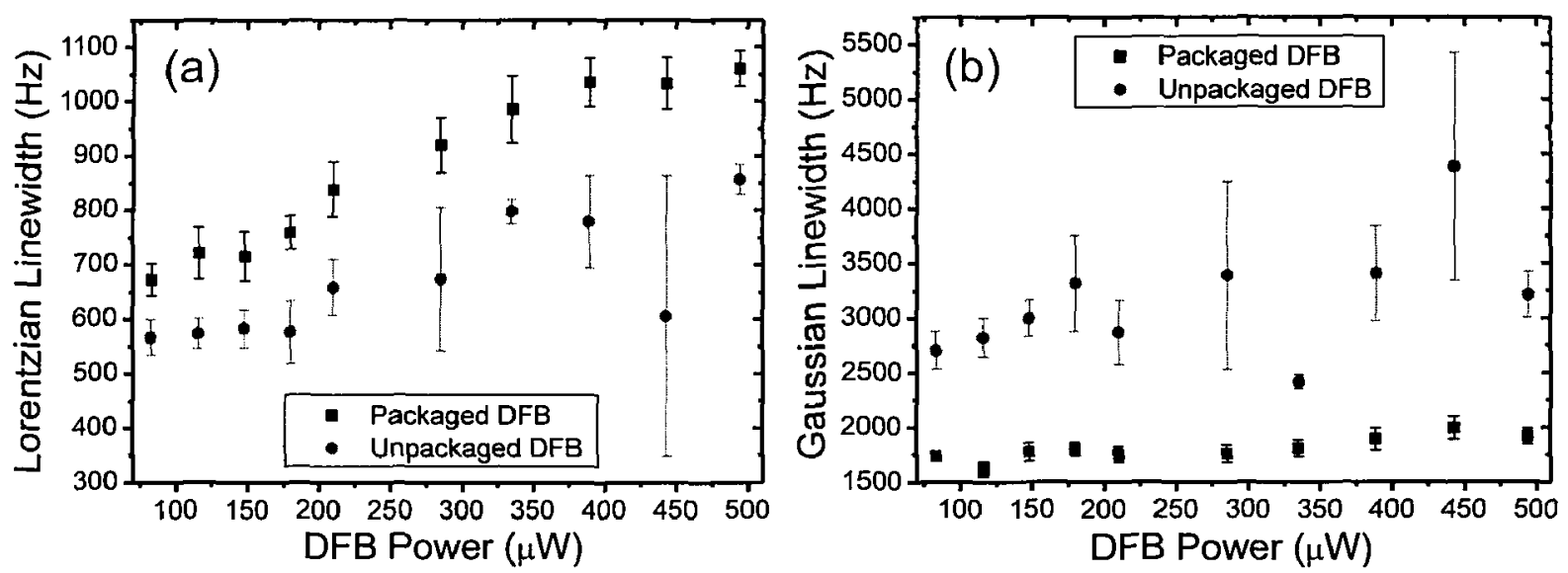

Figure 3 (a) Lorentzian, $w_{\mathrm{L}}$ and (b) Gaussian, $w_{\mathrm{G}}$ linewidths extracted through Voigt fitting of each measurement at a range of DFB powers

\section{CONCLUSIONS}

The MI-DSHI technique was used to compare two identical DFB lasers, one packaged and another unpackaged, to study the impact of packaging. The Lorentzian and Gaussian linewidths were extracted using Voigt fitting of the acquired beat spectra. A significant improvement was observed with packaging, particularly for the Gaussian component which originates primarily from environmental perturbations. The measurement noise was significantly lower for the packaged DFB laser for both the Lorentzian and Gaussian linewidths. By contrast, the Lorentzian linewidth increased in the packaged DFB laser indicating some chirp was possibly introduced to the grating by the packaging process. We suspect the absence of any laser input instability as a result of this, may provide information on the nature of the polarisation compensation introduced during grating writing. Therefore, these measurements provide direct information of relevance to further improving packaging of a DFB fibre laser and in this case some potential insight into the nature of the industrial process used to produce this DFB. Finally, we note that the MI-DSHI has resolved some of the polarisation instabilities that have made such comparative studies difficult using standard MZ-DSHI.

\section{ACKNOWLEDGEMENTS}

Funding from the Australian Research Council (ARC) and Thales Australia Ltd, Underwater Systems Division through Linkage Project LP0776380 is acknowledged. Contributions from ARC DP grants DP0776092, D0879465 are also acknowledged.

\section{REFERENCES}

[1] Ball, G.A., C.G. Hullallen, and J. Livas, Frequency Noise of a Bragg Grating Fiber Laser. Electronics Letters, 1994. 30(15): p. 1229-1230.

[2] Okoshi, T., K. Kikuchi, and A. Nakayama, Novel Method for High-Resolution Measurement of Laser Output Spectrum. Electronics Letters, 1980. 16(16): p. 630-631.

[3] Henry, C.H., Theory of the Linewidth of Semiconductor-Lasers. IEEE Journal of Quantum Electronics, 1982. 18(2): p. 259-264.

[4] Foster, S., A. Tikhomirov, and M. Milnes, Fundamental thermal noise in distributed feedback fiber lasers. IEEE Journal of Quantum Electronics, 2007. 43(5-6): p. 378-384.

[5] Rønnekleiv, E., Frequency and Intensity Noise of Single Frequency Fiber Bragg Grating Lasers. Optical Fiber Technology, 2001. 7(3): p. 206-235. 
[6] Chen, X., M. Han, Y. Zhu, B. Dong, and A. Wang, Implementation of a loss-compensated recirculating delayed self-heterodyne interferometer for ultranarrow laser linewidth measurement. Appl. Opt., 2006. 45(29): p. 7712-7717.

[7] Davis, M.K., M.J.F. Digonnet, and R.H. Pantell, Thermal effects in doped fibers. Journal of Lightwave Technology, 1998. 16(6): p. 1013-1023.

[8] Horak, P., N.Y. Voo, M. Ibsen, and W.H. Loh, Pump-noise-induced linewidth contributions in distributed feedback fiber lasers. IEEE Photonics Technology Letters, 2006. 18(9-12): p. 9981000.

[9] Voo, N.Y., P. Horak, M. Ibsen, and W.H. Loh, Anomalous linewidth behavior in short-cavity single-frequency fiber lasers. IEEE Photonics Technology Letters, 2005. 17(3): p. 546-548.

[10] Foster, S.B. and A.E. Tikhomirov, Pump-Noise Contribution to Frequency Noise and Linewidth of Distributed-Feedback Fiber Lasers. IEEE Journal of Quantum Electronics, 2010. 46(5): p. 734741.

[11]Bedwell, I.R. and D.R. Jones. Fiber laser sensor hydrophone performance. in OCEANS 2010 IEEE - Sydney. 2010.

[12] Mercer, L.B., I/f frequency noise effects on self-heterodyne linewidth measurements. Journal of Lightwave Technology, 1991. 9(4): p. 485-493.

[13] Michie, A. and J. Canning, Properties of a twisted DFB fibre laser, in ACOFT. 2002, Postdeadline paper: Sydney, Australia. p. see patent: J. Canning, A. Michie,et. al. Helical feedback laser operation in a linear DFB laser, PS2846. . 